\title{
Assessment of quality of life in lung transplantation using a simple generic tool
}

\author{
A C Anyanwu, A McGuire, C A Rogers, A J Murday
}

\begin{abstract}
Background-The EuroQol is a generic questionnaire developed to provide a simple method for assigning utility values to health. This study examines the applicability of the EuroQol to the measurement of quality of life in single, bilateral, and heart-lung transplantation.

Methods-A cross sectional study was performed in 87 patients awaiting lung transplantation and in 255 transplant recipients attending follow up clinics in four transplant units.

Results-In the waiting list group 61\% reported extreme problems in at least one of the five EuroQol quality of life domains compared with $20 \%$ single lung recipients, $4 \%$ bilateral lung recipients, and $2 \%$ heart-lung recipients at 3 or more years after transplantation. The mean utility value of patients on the waiting list was 0.31. In comparison, utility values for recipients 3 years after transplantation were 0.61 for single, 0.82 for bilateral, and 0.87 for heart-lung transplants. The utility scores and health profiles of bilateral and heart-lung recipients were consistently superior to those of single lung recipients. Problems in all five domains were more frequent in single lung recipients. Subjective assessment with a visual analogue scale showed a similar trend.

Conclusions-The EuroQol is a simple method of deriving a single utility value for quality of life and is responsive to changes after lung transplantation. It is worth considering as a means of monitoring quality of life after transplantation and as an index of quality of survival in research studies in solid organ transplantation. These data suggest that quality of life after transplantation of one lung is inferior to that after transplantation of two lungs.

(Thorax 2001;56:218-222)
\end{abstract}

Keywords: EuroQol; lung transplantation; quality of life

Intrathoraci

Transplantation, St George's

Hospital, London

SW17 0QT, UK

ajmurday@sghms.ac.uk

Presented at the 19th Annual Meeting of the International Society of Heart and Lung

Transplantation in San

Francisco, April 1999.

Received 25 April 2000

Returned to authors

18 September 2000

Revised version received

6 October 2000

Accepted for publication

4 December 2000 survival benefit on all diagnostic groups undergoing the procedure, ${ }^{2}$ the primary benefit in many patients being an improvement in quality of life. Although the great improvement in quality of life after lung transplantation is well documented $^{34}$ and the importance of quality of life as an outcome measure for lung transplantation is well recognised, ${ }^{5}$ few researchers have used it as an outcome measure. The major barrier to routine measurement of quality of life in clinical and research practice appears to be the difficulty and complexity of measurement. Most existing methods require the use of detailed questionnaires or interviews requiring considerable time investment by researcher and patient. Additionally, the output data are often disaggregated and use ordinal rather than interval scaling, thus restricting the ability for mathematical manipulation. We have measured the quality of life in a cross section of lung transplant patients using a generic tool that is easy to complete and produces an aggregate numerical value for quality of life.

\section{Methods}

EUROQOL QUESTIONNAIRE

Health utility and health related quality of life (HRQoL) were measured using the EuroQol EQ5D questionnaire. ${ }^{67}$ The questionnaire was developed by an international research group primarily to enable the assignment of utility values to health related quality of life. The EQ5D defines health in five dimensions (mobility, self-care, usual activities, pain and discomfort, and anxiety or depression). In each dimension a respondent can belong to one of three categories - no problem, moderate problem, or severe problems. Combinations of these categories result in 243 permutations of health states. A regression equation defines a utility value for these health states. The possible values for health utility ranges from -0.59 (severe problems in all five dimensions) to 1 (no problem in all dimensions) on a scale where 0 represents death and 1 represents the best possible health state. Methods used to derive these utility scores have been described in detail by Dolan. ${ }^{8}$ A utility value is therefore ascribed to an individual's health state based on the absence or presence of moderate or severe problems in the five dimensions. The EQ5D also has a visual analogue scale that enables respondents subjectively to assess their health on a scale ranging from 0 (worst possible health state) to 100 (best possible health state). The EQ5D has been applied in a wide variety of medical specialities and its validity and reliability extensively tested. ${ }^{7}$ No other quality of life tools were used in this study as measure- 
ment of aggregate health utility, rather than specific aspects of quality of life, was the main objective.

ADMINISTRATION OF QUESTIONNAIRES

This study was a multicentre cross sectional questionnaire survey. Patients from four of the seven UK lung transplant centres participated in the study which lasted for 6 months in 1998 . A batch of questionnaires was given to each centre with instructions that all patients attending the lung transplant clinic be given a questionnaire to complete. It was expected that, using this approach, we could obtain a snapshot of all the relevant phases in the preand post-transplant period. All returns were anonymous.

Pre-transplant status was evaluated by administering the EQ5D to waiting list patients in two transplant centres over a 3 month period. It was recognised that patients on the waiting list would be at different stages in their disease and that health status may vary with length of time on the list. For the purpose of this analysis it was assumed that the health status on the waiting list was constant and that any variation in response was a reflection of individual variations in health status rather than of factors related to disease, planned procedure, or duration on the waiting list. EuroQol questionnaires were given to patients attending transplant follow up clinics in four centres. There were no prescribed exceptions: centres were encouraged to give questionnaires to all patients attending their clinics.

STATISTICAL ANALYSIS

Analysis of variance was used to compare mean utility scores between recipients of single, bilateral, and heart-lung transplants and among the

Table 1 Distribution of respondents in the transplanted group by epoch and organ

\begin{tabular}{llllrl}
\hline \multirow{5}{*}{ Organ } & \multicolumn{3}{c}{ Epoch (months after transplant) } & \\
\cline { 2 - 5 } & $0-6$ & $7-18$ & $19-36$ & $>36$ & Total \\
\hline Single & 20 & 20 & 26 & 40 & 106 \\
Bilateral & 14 & 16 & 21 & 28 & 79 \\
Heart-lung & 7 & 7 & 14 & 42 & 70 \\
Total & 41 & 43 & 61 & 110 & 255 \\
\hline
\end{tabular}

Table 2 Percentage of single lung recipients reporting problems in each EuroQol dimension. Data for waiting list patients are shown for comparison.

\begin{tabular}{|c|c|c|c|c|c|}
\hline \multirow[b]{2}{*}{ EuroQol dimension } & \multirow[b]{2}{*}{ Waiting list } & \multicolumn{4}{|c|}{ Epoch (months after transplant) } \\
\hline & & $0-6$ & $7-18$ & $19-36$ & $>36$ \\
\hline \multicolumn{6}{|l|}{ Mobility } \\
\hline Extreme problem & 7 & 0 & 0 & 0 & 0 \\
\hline Any problem & 100 & 35 & 65 & 69 & 83 \\
\hline \multicolumn{6}{|l|}{ Self-care } \\
\hline Extreme problem & 6 & 0 & 0 & 0 & 0 \\
\hline Any problem & 71 & 30 & 20 & 27 & 28 \\
\hline \multicolumn{6}{|l|}{ Usual activities } \\
\hline Extreme problem & 55 & 20 & 20 & 15 & 15 \\
\hline Any problem & 100 & 65 & 80 & 77 & 80 \\
\hline \multicolumn{6}{|l|}{ Pain and discomfort } \\
\hline Extreme problem & 17 & 0 & 0 & 0 & 8 \\
\hline Any problem & 82 & 50 & 70 & 69 & 60 \\
\hline \multicolumn{6}{|l|}{ Anxiety or depression } \\
\hline Extreme problem & 9 & 5 & 0 & 4 & 10 \\
\hline Any problem & 70 & 35 & 30 & 38 & 45 \\
\hline \multicolumn{6}{|l|}{ Any dimension } \\
\hline Extreme problem & 61 & 25 & 20 & 19 & 20 \\
\hline Any problem & 100 & 70 & 95 & 85 & 90 \\
\hline
\end{tabular}

various epochs. The Waller test was used for post hoc comparisons.

\section{Results}

Overall, 87 waiting list and 255 post-transplant questionnaires were used for this analysis. An additional 10 questionnaires returned were discarded because they lacked key information (organ transplanted or date transplanted) necessary for processing of the data.

WAITING LIST (NON-TRANSPLANTED) GROUP Of the 87 patients whose pre-transplant questionnaires were analysed, 28 were listed for a single lung transplant, 24 for a bilateral transplant, and 34 for a heart-lung transplant (type of transplant awaited was missing for one respondent). The mean (SD) age was 39 (14) years. The age distribution was similar to that of patients listed for lung transplantation in the UK. ${ }^{9}$

All waiting list patients reported a problem in at least one EuroQol dimension with $61 \%$ reporting at least one extreme problem. All patients reported problems in performing their usual activities and in mobility. Of the patients listed for single lung transplantation, $75 \%$ (70\% CI 67 to 83 ) reported extreme problems in at least one dimension compared with $50 \%$ (70\% CI 40 to 60 ) for bilateral transplantation and $59 \%(70 \%$ CI 51 to 67$)$ for heart-lung transplantation.

The mean (SD) self-rated health status as recorded by the visual analogue scale was 35 (19) while the mean EuroQol utility score was $0.31(0.31)$.

\section{POST-TRANSPLANTATION}

Of the 255 respondents whose post-transplant data were analysed, 106 had received single lung transplants, 79 bilateral, and 70 heartlung transplants. Post-transplant data from the EuroQol questionnaires were divided into four epochs: 0-6 months, 7-18 months, 19-36 months, and $>36$ months. The distribution of these patients in the four epochs is shown in table 1.

The EuroQol health profiles for the four post-transplant epochs are shown in tables 2-4. Compared with the patients on the waiting list, all three transplant groups showed improvement in all five EuroQol dimensions. In the immediate postoperative period ( $0-6$ months) the proportion of respondents reporting extreme problems in one or more dimensions was $25 \%(70 \%$ CI 15 to 35$)$ of single lung recipients, $7 \%(70 \%$ CI 1 to 14$)$ bilateral lung recipients, and $14 \% \quad(70 \%$ CI 1 to 27$)$ heart-lung recipients compared with $61 \%$ (70\% CI 56 to 66 ) for waiting list respondents. In the longer term there was, however, some difference in quality of life between the transplant groups, the quality of life being better in the bilateral lung and heart-lung recipients than in the single lung recipients. Figure 1 shows the percentage of respondents reporting problems by epoch; at any given period after 6 months $40-60 \%$ of bilateral and heart-lung recipients recorded no problems (in any dimension) compared with $5-15 \%$ in the 
single lung group. The proportion of respondents reporting problems in each dimension after 36 months is shown in fig 2; problems in one or more EuroQol dimensions were reported by $90 \%$ ( $70 \%$ CI 85 to 95$)$ single lung recipients, $61 \%(70 \%$ CI 52 to 70$)$ bilateral lung recipients, and $43 \%$ ( $70 \%$ CI 35 to 51$)$ heart-lung recipients.

Table 3 Percentage of bilateral lung recipients reporting problems in each EuroQol dimension

\begin{tabular}{|c|c|c|c|c|}
\hline \multirow[b]{2}{*}{ EuroQol dimension } & \multicolumn{4}{|c|}{ Epoch (months after transplant) } \\
\hline & $0-6$ & $7-18$ & $19-36$ & $>36$ \\
\hline \multicolumn{5}{|l|}{ Mobility } \\
\hline Extreme problem & 0 & 0 & 0 & 0 \\
\hline Any problem & 43 & 25 & 29 & 46 \\
\hline \multicolumn{5}{|l|}{ Self-care } \\
\hline Extreme problem & 0 & 0 & 0 & 0 \\
\hline Any problem & 7 & 6 & 5 & 11 \\
\hline \multicolumn{5}{|l|}{ Usual activities } \\
\hline Extreme problem & 7 & 0 & 5 & 4 \\
\hline Any problem & 71 & 50 & 50 & 46 \\
\hline \multicolumn{5}{|l|}{ Pain and discomfort } \\
\hline Extreme problem & 0 & 0 & 0 & 0 \\
\hline Any problem & 71 & 50 & 38 & 32 \\
\hline \multicolumn{5}{|l|}{ Anxiety or depression } \\
\hline Extreme problem & 0 & 0 & 0 & 0 \\
\hline Any problem & 14 & 25 & 43 & 29 \\
\hline \multicolumn{5}{|l|}{ Any dimension } \\
\hline Extreme problem & 7 & 0 & 5 & 4 \\
\hline Any problem & 79 & 56 & 57 & 61 \\
\hline
\end{tabular}

Table 4 Percentage of heart-lung recipients reporting problems in each EuroQol dimension

\begin{tabular}{|c|c|c|c|c|}
\hline \multirow[b]{2}{*}{ EuroQol dimension } & \multicolumn{4}{|c|}{ Epoch (months after transplant) } \\
\hline & $0-6$ & $7-18$ & $19-36$ & $>36$ \\
\hline \multicolumn{5}{|l|}{ Mobility } \\
\hline Extreme problem & 0 & 0 & 0 & 0 \\
\hline Any problem & 29 & 29 & 29 & 33 \\
\hline \multicolumn{5}{|l|}{ Self-care } \\
\hline Extreme problem & 0 & 0 & 0 & 0 \\
\hline Any problem & 14 & 0 & 0 & 2 \\
\hline \multicolumn{5}{|l|}{ Usual activities } \\
\hline Extreme problem & 14 & 0 & 0 & 0 \\
\hline Any problem & 57 & 43 & 36 & 31 \\
\hline \multicolumn{5}{|l|}{ Pain and discomfort } \\
\hline Extreme problem & 0 & 0 & 0 & 2 \\
\hline Any problem & 100 & 29 & 43 & 24 \\
\hline \multicolumn{5}{|l|}{ Anxiety or depression } \\
\hline Extreme problem & 0 & 0 & 0 & 0 \\
\hline Any problem & 43 & 43 & 7 & 21 \\
\hline \multicolumn{5}{|l|}{ Any dimension } \\
\hline Extreme problem & 14 & 0 & 0 & 2 \\
\hline Any problem & 100 & 57 & 64 & 43 \\
\hline
\end{tabular}

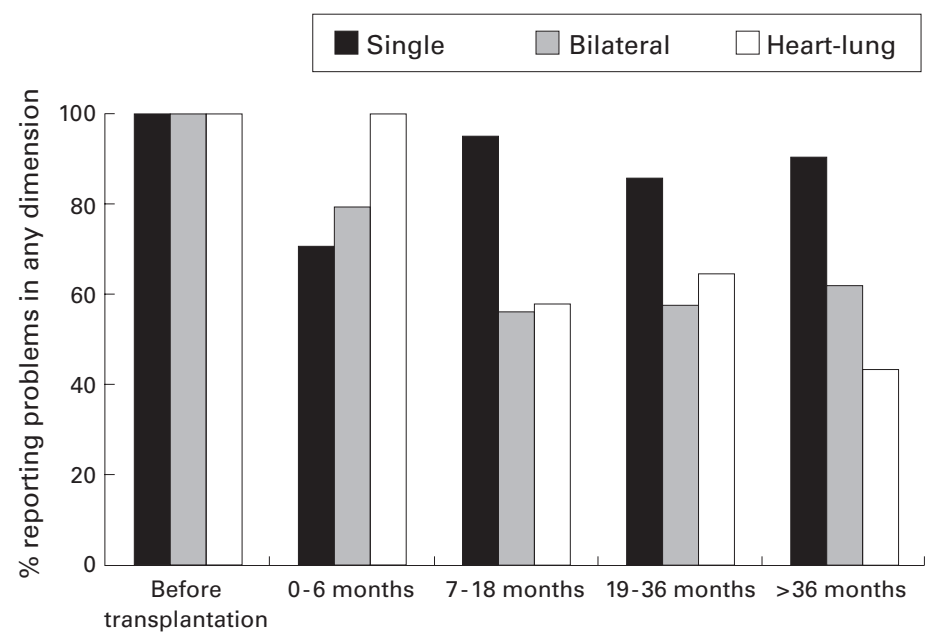

Figure 1 Percentage of respondents reporting problems in one or more EuroQol dimensions at the various epochs.
The EuroQol utility and visual analogue scores for the four post-transplant epochs are presented in table 5. The scores show a substantial improvement in both the EuroQol and visual analogue scores compared with the waiting list group. Analysis of variance showed a significant improvement in health utility after transplantation compared with pre-transplant scores $(p=0.001)$, but no significant differences among the four post-transplant epochs. As with the disaggregated health profiles, the aggregated health status for bilateral and heart-lung recipients was superior to that of the single lung group. In all epochs after 6 months the quality of life, as measured by both the EuroQol score and visual analogue score, was consistently superior in those receiving bilateral and heartlung transplants $(p=0.001)$. There was no significant difference between the bilateral lung and heart-lung groups.

\section{Discussion}

The EuroQol was primarily developed to allow an aggregate value (utility value) to be attached to HRQoL, thus allowing measurement of quality of life as an outcome and enabling weighting of other outcomes (such as survival to produce quality adjusted life-years). Two previous studies that used EuroQol in lung transplantation were identified in the literature. ${ }^{11}$ The study by Busschbach and colleagues ${ }^{11}$ was a pilot study of six patients and only used the visual analogue component of the EuroQol. The other study from the Netherlands ${ }^{10}$ used the EuroQol to derive utility values. The EuroQol has the advantage of being a simple self-completed questionnaire requiring about 1 minute for completion. Health utility has been traditionally measured by time trade off and standard gamble techniques; these methods are interview administered, requiring considerable amount of time, and therefore cannot be applied in routine clinical practice. As improvement of HRQoL is the primary objective of lung transplantation, simple methods of ascribing aggregate values to recipients' quality of life are desirable. The EuroQol also provides disaggregated information on quality of life in five key domains.

This study has shown that quality of life in all five EuroQol domains is better in the transplanted group than in those on the waiting list, which suggests that the EuroQol is responsive to changes in quality of life resulting from symptomatic improvement after lung transplantation. Compared with the $61 \%$ of waiting list respondents who experienced extreme problems in one or more EuroQol dimensions, no bilateral or heart-lung recipients and only $20 \%$ of single lung recipients reported extreme problems 1 year after transplantation. The results are consistent with clinical experience; as expected, all patients on the waiting list reported problems in performing their usual activities with 55\% reporting extreme problems. In contrast, at 3 or more years after transplantation $15 \%$ of single recipients, $4 \%$ of bilateral recipients, and no $(0 \%)$ heart-lung recipients reported extreme limitation in 


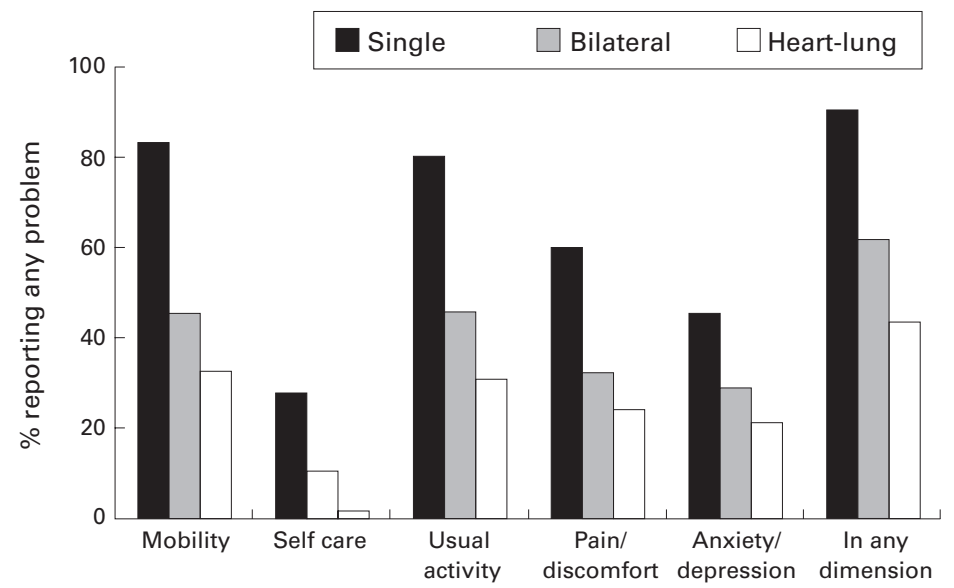

Figure 2 Percentage of respondents more than 36 months after transplantation reporting problems in each EuroQol dimension ( $n=40$ single, 28 bilateral, and 42 heart-lung transplants).

Table 5 Mean (SD) utility and visual analogue scores after pulmonary transplantation.

\begin{tabular}{lllll}
\hline \multicolumn{5}{l}{ Epoch (months after transplant) } \\
\cline { 2 - 4 } & $0-6$ & $7-18$ & $19-36$ & $>36$ \\
\hline Single lung & & & \\
$\quad$ EuroQol score & $0.69(0.31)$ & $0.66(0.21)$ & $0.65(0.24)$ & $0.61(0.31)$ \\
$\quad$ VAS & $67(17)$ & $65(17)$ & $65(20)$ & $60(19)$ \\
Bilateral lung & $0.75(0.17)$ & $0.83(0.17)$ & $0.81(0.19)$ & $0.82(0.19)$ \\
$\quad$ EuroQol score & $79(10)$ & $78(11)$ & $79(18)$ & $77(18)$ \\
$\quad$ VAS & $0.67(0.15)$ & $0.85(0.17)$ & $0.86(0.12)$ & $0.87(0.20)$ \\
$\quad \begin{array}{l}\text { Heart-lung } \\
\quad \text { VuroQol score }\end{array}$ & $76(11)$ & $79(17)$ & $79(13)$ & $79(19)$ \\
\hline
\end{tabular}

VAS = visual analogue score

For the pre-transplant group the mean (SD) utility score was 31 (31) and the visual analogue score was 35 (19).

performing usual activities. The disaggregated results are also in agreement with those reported by earlier workers. ${ }^{3-5} 111^{12}$ Although the EuroQol is a simple tool, its validity and responsiveness is well demonstrated in several medical specialities. ${ }^{7}$ This study adds support to its use in lung transplantation. The aggregate EuroQol utility scores also responded as predicted and were in line with those assigned by Dutch lung transplant recipients in a similar study which also used EuroQol. ${ }^{10}$ As a test of internal validity, the EuroQol scores were compared with the self-rated visual analogue scores and showed good association. The study design made it impossible to record the questionnaire response rate directly as questionnaires were administered by the centres who did not (and were not obliged to) keep a record of the number of questionnaires issued. The centres were not asked to keep a record of the questionnaires issued as that would have created extra work and may have made staff at the centres less inclined to help with data collection. The utility values obtained were, however, similar to those in the Dutch study ${ }^{10}$ which measured utility longitudinally on the same sample. It can therefore be assumed that, despite the limitations of our approach, the cross sectional utility data were a good representation of the true health utility before and after lung transplantation.

The most interesting observation from the quality of life data was that the health profiles and health utility values when one lung was transplanted (single lung) were consistently inferior to the values achieved when two lungs were used (as bilateral or heart-lung transplants). These results were consistent even when examining self-rated visual analogue scores. A review of the literature did not reveal any studies which specifically compared the quality of life in single and bilateral lung recipients. Gartner and colleagues have recently suggested that the utility scores after single and bilateral lung transplantation may not necessarily be the same. ${ }^{13}$ They used the quality of well being scale (another generic instrument) to assess the health status of 20 recipients, which included 16 single lung recipients, and found a mean utility score of 0.60 . They suggested that the preponderance of single lung recipients in their population could have influenced this utility value. Their figure is in line with the 0.61 utility score for single lung recipients found in this study. In contrast, a higher utility score of 0.9 was found in the Dutch cohort. ${ }^{10}$ Unlike the predominance of single lung transplantation in Gartner's cohort, about $80 \%$ of Dutch transplants between 1990 and 1996 were bilateral lung transplants. ${ }^{14}$ This utility score is similar to that reported for bilateral and heart-lung recipients in this study. The high utility scores in the Dutch study (predominantly bilateral) and the low utility score in the study by Gartner et al (predominantly single) support the hypothesis that health status is better after bilateral transplantation than after single lung transplantation.

While the difference in health status after transplantation between recipients of one or two lungs needs to be verified by other studies, plausible biological explanations do exist. The pulmonary physiology in a single lung recipient differs from that in recipients of two lungs. ${ }^{15}$ While most recipients of two lungs achieve their predicted total lung capacity within a year, the same cannot be said of recipients of one lung. Single lung recipients also have less improvement in their forced vital capacity (FVC) and forced expiratory volume $\left(\mathrm{FEV}_{1}\right)$. In addition, the native disease in the contralateral non-transplanted lung can cause physiological and pathological changes. There is therefore a biological basis for believing that single lung transplantation may be less effective than transplantation of two lungs.

There is, however, the possibility of confounding factors as single lung transplantation is primarily reserved for selected diseases (mostly emphysema and pulmonary fibrosis). Patients undergoing single lung transplantation are often older patients with emphysema while those who have bilateral lung grafts are younger patients with cystic fibrosis. The outcome may therefore be partly related to the native disease or age rather than the fact that they received one lung. Indeed, the pre-transplant data from our study suggested that patients listed for single lung transplantation had a poorer health status than those listed for bilateral grafts. However, Bavaria and colleagues ${ }^{16}$ compared the results of single and bilateral lung transplantation in patients with emphysema and found superiority in the $\mathrm{FEV}_{1}$, six minute walk 
test, and other clinical indices in recipients of bilateral lungs compared with those who received a single lung graft. They suggested that, in patients with emphysema (the most common indication for single lung transplantation), bilateral lung transplantation yields better symptomatic relief.

We have shown that it is feasible to obtain an aggregate value for HRQoL in lung transplantation using a simple tool. The ability to assign a single numerical value to $\mathrm{HRQ}$ oL makes it possible to weight outcome measures such as rejection and survival, depending on the recipients' quality of life. Its simplicity means it can be administered to patients as part of a standard clinical assessment, making it practical for use in research studies and routine clinical follow up. This method can be applied to various clinical and research studies to allow a more accurate quantification of the benefits of lung transplantation.

We thank the following centres and centre data coordinators who administered the questionnaires to their patients: Freeman who administered the questionnaires to their patients: Freeman Middlesex (Sheilagh Vidler); Queen Elizabeth Hospital, BirMiddlesex (Sheilagh Vidler); Queen Elizabeth Hospital, Bir-
mingham (Sharon Beer); Wythenshawe Hospital, Manchester (Jane Reader).

Funding: This work was funded by the Department of Health. The views expressed are those of the authors and not necessarly of the Department of Health. This research forms part of an MSc dissertation by AA accepted by City University, London.

1 Michel BC, Van Hout BA, Bonsel GJ. Assessing the benefits of transplant services. Bailliere's Clin Gastroentero 1994;8:411-23.
2 Hosenpud JD, Bennett LE, Keck BM, et al. Effect of diagnosis on survival benefit of lung transplantation for diagnosis on survival benefit of lung transplant

3 Caine N, Sharples LD, Smyth R, et al. Survival and quality of life of cystic fibrosis patients before and after heart-lung transplantation. Transplant Proc 1991;23:1203-4

4 TenVergert EM, Essink BM, Geertsma A, et al. The effect of lung transplantation on health-related quality of life: a longitudinal study. Chest 1998;113:358-64.

5 Gross CR, Raghu G. The cost of lung transplantation and the quality of life post-transplant. Clin Chest Med 1997;18: 391-403.

6 EuroQol Group. EuroQol: a new facility for the measurement of health related quality of life. Health Policy 1990;16: 199-208.

7 Brooks R. EuroQol: the current state of play. Health Policy 1996;37:53-72

8 Dolan P. Modeling valuations for EuroQol health states. Med Care 1997;35:1095-108.

9 Anyanwu AC, Rogers CA, Murday AJ. Variations in cardiac transplantation: comparisons between the United Kingdom and the United States. F Heart Lung Transplant 1999; 18:297-303.

10 Al MJ, Koopmanschap MA, van Enckevort PJ, et al. Cost-effectiveness of lung transplantation in The Netherlands: a scenario analysis. Chest 1998;113:124-130.

11 Busschbach JJ, Horikx PE, van den Bosch JM, et al. Measuring the quality of life before and after bilateral lung ing the quality of life before and after bilateral lung 105:911-7.

12 Dennis C, Caine N, Sharples L, et al. Heart-lung transplantation for end-stage respiratory disease in patients with cystic fibrosis at Papworth Hospital. f Heart Lung Transplant 1993;12:893-902.

13 Gartner SH, Sevick MA, Keenan RJ, et al. Cost-utility of lung transplantation: a pilot study. $\mathcal{F}$ Heart Lung Transplant 1997;16:1129-34.

14 Geertsma A, TenVergert E, Bonsel GJ, et al. Does lung transplantation prolong life? A comparison of survival with and without transplantation. F Heart Lung Transplant 1998; 17:511-6.

15 Girgis RE, Fishman R, Theodore J. Physiology and pharmacology of the transplanted lung. In: Cooper DK, Miller LW, Patterson GA, eds. The transplantation and replacement of thoracic organs. 2nd ed. Dordrecht: Kluwer, 1996: 489-504.

16 Bavaria JE, Kotloff R, Palevsky H, et al. Bilateral versus single lung transplantation for chronic obstructive pulmonary disease. F Thorac Cardiovasc Surg 1997;113:520-7. 